\title{
ON THE LIMIT INFERIOR AND LIMIT
} SUPERIOR FOR DOUBLE SEQUENCES

\section{ÜMIT TOTUR}

Abstract. Let $\left(u_{m n}\right)$ be a double sequence of real numbers such that $\limsup \sigma_{m n}(u)=\beta$ and $\liminf \sigma_{m n}(u)=\alpha$, where $\sigma_{m n}(u)=\frac{1}{(m+1)(n+1)} \sum_{j=0}^{m} \sum_{k=0}^{n} u_{j k}$, and $\alpha \neq \beta$. In this paper, it is presented that $\limsup u_{m n}=\beta$ and $\liminf u_{m n}=\alpha$ if the following conditions hold: For $\lambda>1$

$$
\liminf \frac{1}{([\lambda m]-m)([\lambda n]-n)} \sum_{j=m+1}^{[\lambda m]} \sum_{k=n+1}^{[\lambda n]}\left(u_{j k}-u_{m n}\right) \geqslant 2(\beta-\alpha) \frac{\lambda(2 \lambda-1)}{(\lambda-1)^{2}},
$$

for $0<\lambda<1$

$$
\liminf \frac{1}{(m-[\lambda m])(n-[\lambda n])} \sum_{j=[\lambda m]+1}^{m} \sum_{k=[\lambda n]+1}^{n}\left(u_{m n}-u_{j k}\right) \geqslant 2(\beta-\alpha) \frac{\lambda}{(\lambda-1)^{2}},
$$

where $[\lambda n]$ denotes the integer part of $\lambda n$.

Mathematics subject classification (2010): 40A05, 40G05, 40E05.

Keywords and phrases: Double sequences, limit inferior and limit superior, convergence in Pringsheim's sense.

\section{REFERENCES}

[1] İ. ÇANAK, An extended Tauberian theorem for the $(C, 1)$ summability method, Appl. Math.Lett. 21, 1 (2008), 74-80.

[2] İ. ÇANAK, On (C,1) means of sequences, Comput. Math. Appl. 62, 9 (2011), 3446-3448.

[3] İ. ÇANAK, A theorem on the Cesàro summability method, Comput. Math. Appl. 61, 4 (2011), 1162 1166.

[4] İ. ÇANAK, Ü. TotUR, Some Tauberian conditions for Cesàro summability method, Math. Slovaca 62, 2 (2012), 271-280.

[5] İ. ÇANAK, Ü. TOTUR, A condition under which slow oscillation of a sequence follows from Cesàro summability of its generator sequence, Appl. Math. Comput. 216, 5 (2010), 1618-1623.

[6] K. KNopP, Limitierungs-Umkehrsätze für Doppelfolgen, Math. Z. 45, (1939), 573-589.

[7] F. MóRICZ, Tauberian theorems for Cesàro summable double sequences, Stud. Math. 110, 1 (1994), $83-96$.

[8] M. Mursaleen, F. BAŞAR, Domain of Cesàro mean of order one in some spaces of double sequences, Studia Sci. Math. Hungar. 51, 3 (2014), 335-356.

[9] R. F. Patterson, Double sequence core theorems, Int. J. Math. Math. Sci. 22, 4 (1999), 785-793.

[10] A. Pringsheim, Zur Theorie der zweifach unendlichen Zahlenfolgen, Math. Ann. 53, (1900), 289321.

[11] Ü. Totur, Classical Tauberian theorems for the $(C, 1,1)$ summability method, An. Ştiinţ. Univ. Al. I. Cuza Iaşi. Mat. 61, 2 (2015), 401-414. 